(C) The Authors

\title{
An experimental test of the weak equivalence principle for antihydrogen at the future FLAIR facility
}

\author{
Klaus Blaum \\ Max Planck Institute for Nuclear Physics \\ Saupfercheckweg 1, 69117 Heidelberg, Germany \\ klaus.blaum@mpi-hd.mpg.de \\ Mark G. Raizen \\ The University of Texas at Austin, Department of Physics \\ 1 University Station C1600, Austin, TX 78712, USA \\ raizen@physics.utexas.edu \\ Wolfgang Quint* \\ GSI Helmholtzzentrum für Schwerionenforschung \\ Planckstr. 1, 64291 Darmstadt, Germany \\ w.quint@gsi.de
}

Received 19 March 2014

Revised 20 March 2014

Published 7 May 2014

\begin{abstract}
We present new experimental ideas to investigate the gravitational interaction of antihydrogen. The experiment can first be performed in an off-line mirror measurement on hydrogen atoms, as a testing ground for our methods, before the implementation with antihydrogen atoms. A beam of hydrogen atoms is formed by launching a cold beam of protons through a cloud of trapped electrons in a nested Penning trap arrangement. In the next step, the atoms are stopped in a series of pulsed electromagnetic coils so-called atomic coilgun. The stopped atoms are confined in a magnetic quadrupole trap and cooled by single-photon laser cooling. We intend to employ the method of Raman interferometry to study the gravitational interaction of atomic hydrogen — and later on antihydrogen at the FLAIR facility — with high sensitivity.
\end{abstract}

Keywords: Antihydrogen; gravitation; Raman interferometry.

This is an Open Access article published by World Scientific Publishing Company. It is distributed under the terms of the Creative Commons Attribution 3.0 (CC-BY) License. Further distribution of this work is permitted, provided the original work is properly cited.

*Presented by W. Quint. 


\section{Introduction: FLAIR Facility}

FAIR, the Future Accelerator Facility for Beams of Ions and Antiprotons at Darmstadt, will produce the highest flux of antiprotons in the world. ${ }^{1}$ Within the planned complex of storage rings, it will be possible to decelerate the antiprotons to $30 \mathrm{MeV}$ kinetic energy, opening up the possibility to also create low-energy antiprotons for delivery to FLAIR (Facility for Low-energy Antiproton and Ion Research). ${ }^{2}$ The FLAIR physics program comprises laser and microwave spectroscopy on antihydrogen, antiprotonic atom spectroscopy, gravitation of antimatter, interaction of antimatter with matter, and nuclear and particle physics with antiprotons. Here we focus on an experimental concept to investigate the gravitational interaction of antihydrogen. ${ }^{3}$

\section{Motivation and Overview}

The quest to create and study antihydrogen has stimulated an intense effort in recent years. The main motivations for this work are the prospects for testing CPT symmetry at an unprecedented level, and for testing the weak equivalence principle with antimatter. The study of antihydrogen is a grand challenge that has also sparked great public interest and excitement. The starting point for this work is trapping and cooling of antiprotons in cryogenic Penning traps, and great progress has been made. Antihydrogen atoms were created by merging antiprotons with positrons, and were observed by the annihilation signals on adjacent detectors. ${ }^{4,5}$ Despite this impressive progress, serious hurdles remain. One of the problems with the current approach is that the antihydrogen atoms must be trapped in-situ. This requires the combination of a magnetic trap for the neutrals, superimposed on the cryogenic Penning traps. ${ }^{6,7}$ The lack of optical access and the cryogenic environment with superconducting magnets also pose serious impediments to future progress. Finally, the constraints of limited beam time at an accelerator lab make it very difficult to test and implement new concepts. We propose here a new experimental scheme that may overcome the current obstacles, and enable a breakthrough in the long-term quest to study antihydrogen. We suggest to first build an off-line mirror experiment with matter, as a testing ground for our methods. This will bring together new approaches to trapping and cooling of charged particles with general methods of trapping and cooling of neutral atoms. The mirror experiment will serve as a unique test bed which will enable the demonstration of all the required steps. The mirror system will also enable off-line tests of new concepts, and will therefore enable rapid progress with antihydrogen. The basic strategy of the mirror system is to trap and cool protons and electrons in a cryogenic Penning trap. The protons will then be launched to form a beam of neutral hydrogen atoms, which will be stopped and cooled. We now describe in more detail each stage of the planned setup. 


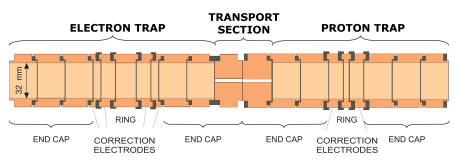

Fig. 1. (Color online) Cryogenic double-Penning-trap system for the simultaneous storage of electrons and protons.

\section{Charged Particle Storage and Cooling}

An ideal trap configuration for the study of the hydrogen formation is a double Penning trap system (see Fig. 1) hosted in the same homogeneous region of one superconducting magnet, enabling a fast and efficient transport of the species under consideration from one trap to the other. ${ }^{8}$ Access for laser beams through windows or fibres is foreseen.

Cooling of positively charged particles in Penning traps can be accomplished by sympathetic cooling. ${ }^{9}$ In this approach, another ion species is confined in the same trap, and is laser-cooled. Coulomb interaction between the particles then cools the desired species. Ionic species that are convenient for laser cooling are $\mathrm{Be}^{+}$and $\mathrm{Mg}^{+}$and they can be used for sympathetic cooling of subatomic particles, such as protons. Sympathetic cooling of antiprotons would require laser cooling of negative ions that are confined in the same trap. However, recent work by A. Kellerbauer et al. on negative osmium ions ${ }^{10}$ showed that direct laser cooling of negative ions is not promising.

An alternative method of reducing the kinetic energy of trapped particles is evaporative cooling, routinely used on neutral atoms confined in magnetic traps. Evaporative cooling of charged particles was employed for Penning-trap mass measurements of highly charged ions at SMILETRAP. ${ }^{11-13}$ The energetic ions are removed from the trap by lowering the trapping potential, and collisions cause the remaining ions to re-equilibrate at a lower temperature. This procedure can be continued until a certain ion temperature is reached. Direct evaporative cooling of antiprotons is not efficient, since it would lead to a huge loss in number. We will, instead, investigate the prospect of sympathetic evaporative cooling using negative ions. In a first step, resistive cooling or electronic feedback cooling (see below) would get the antiprotons to $4 \mathrm{~K}$ and below. Negative ions will then be cooled by evaporation, which can be conveniently done in this case by tuning of a photo-detachment laser. This technique will be tested in the Heidelberg group on a range of negative ion species. We can produce large quantities of, e.g., $\mathrm{H}^{-}, \mathrm{C}^{-}, \mathrm{O}^{-}, \mathrm{Os}^{-}$, etc. as listed in Table 1.

Most of them are produced using a Middleton-type sputter negative-ion source ${ }^{14}$ where beams ranging from a few $\mu \mathrm{A}\left(\mathrm{Os}^{-}\right)$up to a few $100 \mu \mathrm{A}\left(\mathrm{C}^{-}\right)$can be produced. Using a dipole-mass separator magnet with a resolving power of a few 100 at $\sim 5 \mathrm{keV}$ ion energy, high purity negative ion beams can be obtained and efficiently injected into a Penning trap resulting in $10^{4}-10^{6}$ trapped negatively charged ions. 
Table 1. Negative ion species produced at MPI-K Heidelberg with a Middleton-type sputter ion source.

\begin{tabular}{ccc}
\hline Negative ion species & Typical ion current $(\mu \mathrm{A})$ & Maximum ion current $(\mu \mathrm{A})$ \\
\hline $\mathrm{H}^{-}$ & $30-60$ & 130 \\
$\mathrm{C}^{-}$ & 200 & 400 \\
$\mathrm{O}^{-}$ & 100 & 250 \\
$\mathrm{Si}^{-}$ & 100 & 330 \\
$\mathrm{Cl}^{-}$ & 50 & 90 \\
$\mathrm{Cu}^{-}$ & 50 & 100 \\
$\mathrm{Se}^{-}$ & 40 & 60 \\
$\mathrm{Os}^{-}$ & 3 & 10 \\
$\mathrm{Au}^{-}$ & 30 & 100 \\
\hline
\end{tabular}

\section{Detection}

Highly-sensitive and non-destructive detection is needed in order to measure the tiny currents $(\sim 1-100 \mathrm{fA})$ induced in the trap electrodes by the oscillating particles. The techniques described in the following have been employed for precision measurements of atomic and nuclear ground state properties such as masses and magnetic moments. ${ }^{15-20}$ The amplitudes of the ion motion must be kept small to avoid heating of the particles and to minimize the influence of imperfections of the trapping fields. The resonator and the amplifier have to work at cryogenic temperatures $(4 \mathrm{~K})$ and in strong magnetic fields $(2-7 \mathrm{~T})$, due to Johnson and electronic noise. This prohibits the use of a wide range of electronic components, including most silicon-based semiconductors. Since the detection system has to be extremely sensitive, highly-tuned circuits of high quality factor ( $Q$-value) must be used, which act in resonance as a high real resistance $R=Q \omega L$, where $\omega$ is the resonance frequency and $L$ the inductance of the tuned circuit. Thus, the tiny single particle current leads to a detectable voltage drop at the amplifiers input, which is mostly based on low-noise GaAs field-effect transistors. State-of-the-art amplifiers have an equivalent input noise of typically $>1 \mathrm{nV} / \mathrm{Hz}^{\frac{1}{2}}$ at the axial frequency of the stored (anti)protons (a few $100 \mathrm{kHz}$ to $1 \mathrm{MHz}$ ). We developed ultralow noise transistor amplifiers based on GaAs MESFETs in combination with novel electronic technology, which led to an electronic noise of $<300 \mathrm{pV} / \mathrm{Hz}^{\frac{1}{2}}$ at $1 \mathrm{MHz}$. This development may enable the application of novel techniques as, e.g., electronic feedback cooling in order to gain access to the millikelvin range for the axial mode temperature during measurements.

The development of active input-impedance compensation techniques allows the use of large gate structures for the front-end transistors with accordingly lower generation and recombination noise. This also reduces white noise densities and gives smaller loading of the attached tank circuit, leading to much higher $Q$ values. ${ }^{21}$ The advantages of GaAs-based FET transistors with those of novel technologies (such as Si:Ge heterostructure bipolar transistors) can push the particle-motion detection frontier. In the context of the antihydrogen project, a large number of 
antiprotons/positrons will be stored. In this regime, feedback cooling works on the fluctuations, and is known in high energy physics as stochastic cooling. Further studies must be performed on protons/electrons in order to test the viability of this approach.

\section{Neutral Atom Experiments}

Trapping and cooling of atoms in the gas phase has been a major area of research for over thirty years. The advances in this field were enabled by laser cooling. Despite the enormous success of this method, it has been limited to a small set of atoms in the periodic table. The reason for the limited applicability of laser cooling is that it requires a two-level cycling transition and one that is accessible with stabilized lasers. These constraints have excluded most of the periodic table as well as any molecules. In particular, laser cooling of hydrogen has not been possible. Magnetic trapping and evaporative cooling of hydrogen was accomplished, ${ }^{22}$ but required a complex dilution refrigerator, and could not be extended to D or T.

In the past few years, there has emerged a simple two-step approach to trapping and cooling that will work on any paramagnetic atom or molecule. This includes most of the periodic table as well as certain molecules. ${ }^{23}$ The first step, the atomic coilgun, uses pulsed magnetic fields to stop atoms. The second step, single-photon cooling, uses a one-way wall to cool the atoms. These new approaches are currently being refined and applied to hydrogenic atoms, H, D, and T. The same methods will also work for antihydrogen (H-bar) and may enable a breakthrough in fundamental tests with neutral antimatter.

We briefly review the principles of the atomic coilgun and single-photon cooling. The typical starting point for these experiments is the supersonic molecular beam which has been the workhorse of physical chemistry for many years, creating a very monochromatic but fast beam of atoms or molecules. These beams are typically operated with a high-pressure noble gas carrier gas that is "seeded" with another gas. Alternatively, atoms or molecules are entrained into the flow near the output of the supersonic valve.

It was proposed that paramagnetic atoms in the supersonic beam could be stopped using a series of pulsed electromagnetic coils. ${ }^{24}$ Most atoms in the periodic table have a magnetic moment in their ground state or in a long-lived metastable state allowing the control of the atomic motion using magnetic fields. The principle of magnetic deceleration is conceptually simple: low-field seekers lose kinetic energy by moving into the high magnetic field region at the centre of an electromagnetic coil. When the atom reaches the top of the magnetic "hill" the magnetic field is suddenly switched off. Due to conservation of energy, the amount of the kinetic energy lost is equal to the Zeeman energy shift, $\Delta \mathrm{E}=\mathrm{g}_{J} \mu_{B} \mathrm{M}_{J} \mathrm{H}$, where $\mathrm{g}_{J}$ is the Landé factor, $\mu_{B}$ is the Bohr magneton, $M_{J}$ is the projection of the total angular momentum on the quantization axis, and $\mathrm{H}$ is the magnetic field. In the ideal operation of the atomic coilgun, the velocity distribution of the atoms is not changed, 
but the mean velocity in the laboratory frame is removed. This is therefore not a cooling process, simply a translation in velocity space.

After stopping the atoms, they can be confined in a magnetic trap. The coilgun has been implemented experimentally and a beam of metastable neon, as well as a beam of molecular oxygen, has been stopped..$^{25-27}$ In parallel work, the same was accomplished for a beam of atomic hydrogen. ${ }^{28-30}$

The recent implementation of an adiabatic atomic coilgun is an important advance over earlier work as it proved that atom-number and phase-space density could be preserved throughout the deceleration process. ${ }^{31,32}$

The next step is to cool the atoms further, and this is where the method of single-photon cooling takes over. The basic construction is cooling with a one-way barrier for atoms, as first proposed in 2005. ${ }^{33}$ The experimental implementation was carried out using a hybrid magnetic and optical trap with atomic rubidium. ${ }^{34} \mathrm{~A}$ phase space enhancement of 350x was reported, although less than $1 \%$ of the atoms were captured in an optical tweezer. ${ }^{35} \mathrm{~A}$ new version of singlephoton cooling is an all-magnetic approach with dressed RF states and a single laser beam to induce the irreversible step. ${ }^{36}$ This version will enable trapping of nearly all the atoms, limited only by the branching ratio of the spontaneous emitted photons (around $50 \%$ ). This method should work well on hydrogen and antihydrogen. In that case, a laser at $243 \mathrm{~nm}$ will drive the two-photon $1 \mathrm{~S}-2 \mathrm{~S}$ transition which will be quenched to emit a Lymanalpha photon at $121 \mathrm{~nm}$.

The same methods of trapping and cooling also naturally lend themselves to precision measurement, as the atoms can be transported into a region that has excellent optical access and is shielded from external magnetic fields. The most sensitive measurement of gravity to date has been accomplished with a Raman Interferometer first developed by Kasevich and Chu in the early nineties. ${ }^{37}$ The principle of the interferometer is to use two ground state hyperfine levels of an atom and to drive two-photon stimulated Raman transitions between those states by tuning the frequency difference between the lasers to match the hyperfine splitting. The configuration of counter-propagating beams for the Raman transition maximizes Doppler sensitivity, and the use of $m=0$ magnetic sublevels minimizes the effect of stray magnetic fields. The geometry for gravity measurements is such that the two Raman beams are aligned with the direction of gravity, and has enabled an absolute measurement of $\mathrm{g}$ to one part per billion. A relative measurement of two isotopes (such as $\mathrm{Rb}-85$ and $\mathrm{Rb}-87$ ) can test the weak equivalence principle for matter, and has the potential for much higher sensitivity because many common systematic cancel.

We propose to apply Raman interferometry to hydrogen and antihydrogen. The first step is to excite the $1 \mathrm{~S}$ state with a two-photon transition at $243 \mathrm{~nm}$ to drive the atoms to the metastable $2 \mathrm{~S}$ state. The lifetime of that state $(120 \mathrm{~ms})$ is long enough to enable a precision measurement. The Raman beams will be tuned about $20 \mathrm{GHz}$ from the 2S-3P transition near $657 \mathrm{~nm}$ (Balmer line). A sequence of three pulses will split and recombine the atomic wavepackets in the direction of gravity, using the 2S hyperfine splitting of $177 \mathrm{MHz}$. The sensitivity of the Raman interferometer 
depends on the accumulated phase shift during the free propagation time $\mathrm{T}$ between pulses, and scales as $\mathrm{T}^{2}$, which emphasizes the importance of an ultracold sample to enable long interaction times. The signal-to-noise scales with the inverse square root of the number of atoms, so is relatively insensitive as compared with the interaction time. A comparative measurement of g-bar/g with this method could be at the level of one part per billion or better. As noted earlier, this measurement could be first demonstrated with $\mathrm{H}$ thereby proving the capability with $\mathrm{H}$-bar. A trapped sample of H-bar also lends itself naturally to a precision spectroscopic measurement of the 1S-2S transition. Further, the atoms can be trapped in a laser tweezer near $515 \mathrm{~nm}$ inside a power build-up cavity to hold the atoms as they are being probed. This wavelength ensures that the optical dipole shifts of the $1 \mathrm{~S}$ and $2 \mathrm{~S}$ states are identical, a so-called magic wavelength that is used in atomic lattice clocks. A spectroscopic comparison between $\mathrm{H}$ and $\mathrm{H}$-bar could ultimately be done at the part in $10^{18}$ level.

\section{Synthesis of the Work: From Hydrogen to Antihydrogen}

The goals of the mirror experiment are to generate a cold beam of hydrogen which will then be stopped and cooled. This will require a complete synthesis of the methods described above. To form a beam, protons will be launched through an electron cloud, forming highly excited Rydberg states of hydrogen. Recent theory has shown that trapped antihydrogen atoms will undergo significant translational cooling as they cascade down to the internal ground state in a strong magnetic field. ${ }^{38,39}$ This same mechanism could be used to cool the beam of hydrogen (and antihydrogen) as they are launched out of the Penning trap, and would require a magnetic field minimum along the axis of propagation. This mechanism will be studied and optimized in the mirror experiment with hydrogen. The beam will be detected using the adiabatic atomic coilgun which will stop the atoms. The timing in this case is provided by the bias voltages on the Penning trap that will launch the protons. Detection of the trapped atoms will be accomplished by laser excitation to the $2 \mathrm{~S}$ state. The atoms can then be launched towards an MCP and detected with near unit efficiency. To test the recombination and beam formation processes, we will use sympathetic cooling of the protons with laser-cooled magnesium ions. To test cooling strategies of charged particles that would be closest to the antihydrogen case, we will use sympathetic evaporative cooling of negative hydrogen ions $\mathrm{H}$ by another negative ion species (see Table 1).

\section{Acknowledgments}

This work was supported by the Max Planck Society, the EU (ERC grant number 290870; MEFUCO), the IMPRS-QD, GSI/FAIR and the Helmholtz Alliance HA216/EMMI. MGR acknowledges support from the US National Science Foundation. 


\section{References}

1. https://www.gsi.de/en/research/fair.htm

2. E. Widmann, Act. Phys. Pol. 41, 249 (2010).

3. K. Blaum and M. G. Raizen, White Paper to FLAIR at GSI, 2009.

4. M. Amoretti et al., Nature 419, 456 (2002).

5. G. Gabrielse et al., Phys. Rev. Lett. 89, 213401 (2002).

6. G. B. Andresen et al., Nature 468, 673 (2010).

7. G. Gabrielse et al., Phys. Rev. Lett. 108, 113002 (2012).

8. W. Quint, R. Kaiser, D. Hall and G. Gabrielse, Hyp. Int. 76, 181 (1993).

9. D. J. Larson et al., Phys. Rev. Lett. 57, 70 (1986).

10. U. Warring et al., Phys. Rev. Lett. 102, 043001 (2009).

11. I. Berström et al., Nucl. Instrum. Meth. A 487, 618 (2002).

12. I. Berström et al., Eur. Phys. J. D 22, 41 (2003).

13. Sz. Nagy et al., Phys. Rev. Lett. 96, 163004 (2006).

14. R. Middleton, Nucl. Instrum. Meth. 214, 139 (1983).

15. K. Blaum, Phys. Rep. 425, 1 (2006).

16. K. Blaum, Sz. Nagy and G. Werth, J. Phys. B 42, 154015 (2009).

17. S. Ulmer et al., Phys. Rev. Lett. 106, 253001 (2011).

18. A. Wagner et al., Phys. Rev. Lett. 110, 033003 (2013).

19. A. Mooser et al., Phys. Rev. Lett. 110, 140405 (2013).

20. S. Sturm et al., Nature 506, 467 (2014).

21. J. Repp et al., Appl. Phys. B 107, 983 (2012).

22. H. Hess et al., Phys. Rev. Lett. 59, 672 (1987).

23. M. G. Raizen, Science 324, 1403 (2009).

24. E. Narevicius et al., New J. Phys. 9, 96 (2007).

25. E. Narevicius et al., New J. Phys. 9, 358 (2007).

26. E. Narevicius et al., Phys. Rev. Lett. 100, 093003 (2008).

27. E. Narevicius et al., Phys. Rev. A 77, 051401 (2008).

28. N. Vanhaecke et al., Phys. Rev. A 75, 031402 (R) (2007).

29. S. D. Hogan et al., Phys. Rev. A 76, 023412 (2007).

30. S. D. Hogan et al., Phys. Rev. Lett. 101, 143001 (2008).

31. E. Lavert-Ofir et al., New J. Phys. 13, 103030 (2011).

32. E. Narevicius and M. G. Raizen, Chem. Rev. 112, 4879 (2012).

33. M. G. Raizen et al., Phys. Rev. Lett. 94, 053003 (2005).

34. G. N. Price et al., Phys. Rev. Lett. 100, 093004 (2008).

35. S. T. Bannerman et al., New Jour. Phys. 11, 063044 (2009).

36. E. Narevicius, S. T. Bannerman and M. G. Raizen, New Jour. Phys. 11, 055046 (2009).

37. M. Kasevich and S. Chu, Phys. Rev. Lett. 67, 181 (1991).

38. C. Taylor, J. Zhang and F. Robicheaux, J. Phys. B 39, 4945 (2006).

39. T. Pohl et al., Phys. Rev. Lett. 97, 213001 (2006). 